\title{
NEW DESIGN OF SYNERGISTIC WRIST SPLINT
}

\author{
Pei-Hsi Chou, You-Li Chou*, Gwo-Feng HuanG*, \\ GAU-TYAN LIN, KUN-I YANG*, FONG-CHIN SU*
}

\begin{abstract}
Department of Orthopedic Surgery, Kaohsiung Medical University, Kaohsiung, *Institute of Biomedical Engineering, National Cheng Kung University, Tainan, Taiwan
\end{abstract}

\begin{abstract}
Tendons are frequently injured by direct trauma. Tendon adhesions are a common factor compromising the results of tendon repair and to this day represent one of the most challenging problems in hand surgery. Use of controlled motion splints during the early stages of tendon recovery increases tendon gliding. Thus, a variety of controlled motion splints have been developed. This paper introduces a new controlled motion splint called the Synergistic Wrist Motion Splint (SWIMS). The "seqential quadratic programming method" was used to optimize Yang's synertistic model of the wrist/hand system, with the results being embodied in a practical controlled motion splint. Derivation of the model is discussed in detail. The dimensions of a patient's hand are input into a general formula to generate a specific SWIMS configuration for the patient. Five prototype SWIMS splints were produced and tested on 5 normal subjects. A three-dimensional motion analysis system was employed to measure the relative motion between the wrist and finger joints due to passive muscle tension for the prototype SWIMS splints, and the results are compared with the mathematical simulation or model.
\end{abstract}

Biomed Eng Appl Basis Comm, 2001 (April); 13: 72-78.

Keywords : Splint, Dynamic Splint, Synergistic Wrist Splint

\section{INTRODUCTION}

Tendon injury is typically the result of direct trauma because the tendon is the medium that transmits force from muscle to bone during motion. Rupture and tendon adhesions are primary problems for flexor tendon recovery, and optimal postoperative treatment for obtaining good motion and strength of repaired flexor tendons has long been a clinical issue. Bunnell (1944) advised that splints should be used for

Received: Jan. 3, 2001; accepted: Feb. 20, 2001.

Correspondence: You-Li Chou, Professor

Institute of Biomedical Engineering,

National Cheng Kung University

No. 1, Ta-Hsueh Road, Tainan, Taiwan

E-mail: ylchou@mail.ncku.edu.tw three to four weeks to protect tendons from breaking during recovery [1]. Mason and Allen (1941) [2] proved that tendons are virtually without strength during the first 5 to 7 days of recovery, and that the tensile strength improves after 14 days. It was found that early motion avoided tendon adhesions. It was also found, however, that early motion caused scar formation at both ends of the tendon. Thus early motion came to be discouraged therapeutically, a concept which limited improvements of wrist rehabilitation for many years. In 1960, Harmon and Young [3] suggested that passive motion with protective splinting would increase tendon gliding and improve functional results. Following this, the concept of "controlled motion" gradually replaced total immobilization. The efficacy of controlled motion has been supported by numerous animal studies performed over the past ten year $[4,5]$. Study of the effects of passive motion on subjects was conducted in a series of in vitro experiments which evaluated and 
compared the differences in tendon excursion between profundus and superficial using several mobilization techniques [6-9]. In these experiments, the concept of "the dynamic splint" was established and evaluated.

The synergistic relationship [9] between wrist joint and finger joint was studied by C. H. Yang (1993) [10]. Our group's attempt at improved splint design began with the premise that consideration of anatomical constraints and the synergistic relationship could aid optimum dynamic wrist splint design. The "seqential quadratic programming method" [14] was used to optimize Yang's model of the wrist/hand system. We found we could embody the optimized solution in a practical controlled motion splint, the Synergistic Wrist Motion Splint (SWIMS). The Type I SWIMS is a six-bar linkage mechanism, as shown in Fig. 1.

This paper will deal with the mathematical derivation of the proposed splint. Prototypes of the Type I SWIMS have been produced and tested on normal subjects. The results will be discussed below.

\section{METHOD}
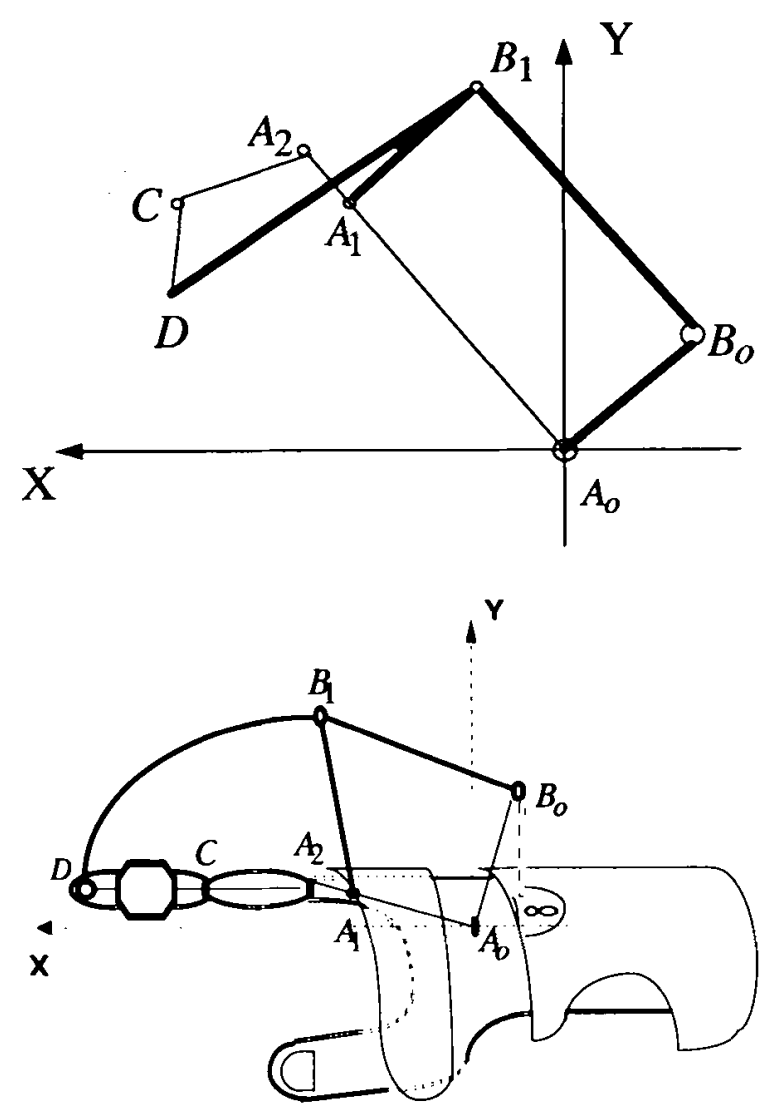

Fig 1. Sketch of Watt's six-bar linkage and of Type I dynamic splint
The Type I SWIMS design is based on Watt's sixbar linkage mechanism [11] (Fig. 2). A structural sketch of the Type I mechanism is shown in Fig. 1.

$$
\begin{aligned}
& A_{o} B_{o}: \text { fixed link (crank 1) } \\
& A_{o} A_{2}: \text { input link (crank 2) } \\
& A_{1} B_{1} D: \text { the connecting link (crank 3) } \\
& B_{o} B_{1}: \text { (crank 4) } \\
& A_{2} C: \text { (crank 5) } \\
& C D: \text { (crank 6) }
\end{aligned}
$$

and we define:

$A_{o} A_{2}$ : the length from Wrist joint to metatarsal phalangel (MP) joint

$A_{2} C$ : the length from MP joint to proximal interphalangel (PIP) joint

\section{$C D$ : the length from PIP joint to PIP joint}

Based on the theory of optimization, we can find the upper and lower bounds of the crank angles and lengths. To do so, first, we set the variables $x_{1}, x_{2}, x_{3}$ and $x_{4}$ as the lengths of crank $r_{1}, r_{3}, r_{4}$ and $I_{5}$ respectively. The curve generated by the possible locations of point $D$ (the tip of the finger, henceforth TIP) is the desired solution. The coordinant points for possible D are thus (Eqs. 1-2):

$$
\begin{aligned}
& f_{D x}=r_{1} \cos \theta_{1}+r_{4} \cos \theta_{4}+r_{5} \cos \theta_{5} \\
& f_{D y}=r_{1} \sin \theta_{1}+r_{4} \sin \theta_{4}+r_{5} \sin \theta_{5}
\end{aligned}
$$

Because the six-bar linkage is a closed loop, the set of possible coordinant points for the position of TIP is (Eqs. 3-4):

$$
\begin{aligned}
& f_{\text {TIPx }}=r_{2} \cos \theta_{2}+r_{6} \cos \theta_{6}+r_{7} \cos \theta_{7} \\
& f_{\text {TIPy }}=r_{2} \sin \theta_{2}+r_{6} \sin \theta_{6}+r_{7} \sin \theta_{7}
\end{aligned}
$$

Based on this, we can find the objective function,

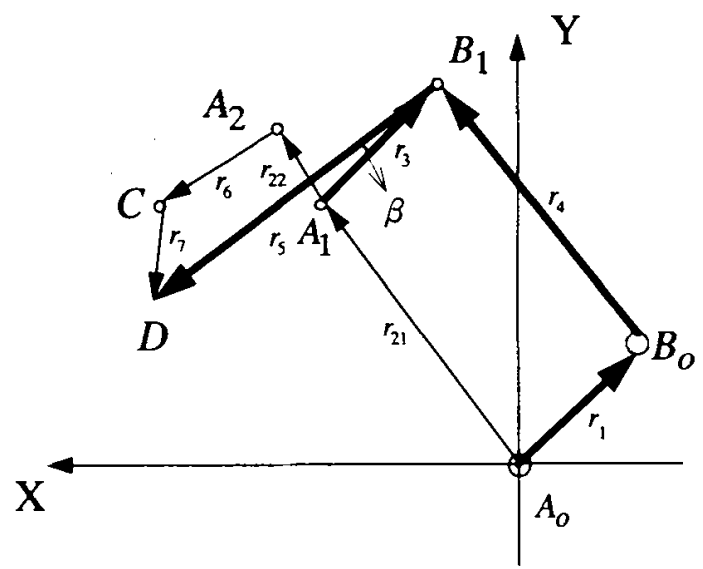

Fig 2. Structural sketch of Watt's six-bar linkage mechanism 
constraint function and the variable of the mechanism by solving these equations via optimization.

$f(X)$ (objective function) (Eqs. 5):

$f(X)=\left(f_{D x}-f_{T P x}\right)^{2}+\left(f_{D x}-f_{\text {II }}\right)^{2}$

$g_{j}(X)$ (constrain function) (Eqs. 6-17):

$g_{1}(X)=-r_{1} \leq 0$

$g_{2}(X)=-r_{3} \leq 0$

$g_{3}(X)=-r_{4} \leq 0$

$g_{4}(X)=-r_{5} \leq 0$

$g_{s}(X)=-\theta_{3} \leq 0$

$g_{6}(X)=-\theta_{4} \leq 0$

$g_{7}(X)=\theta_{3}-\pi / 2 \leq 0$

$g_{8}(X)=\theta_{4}-3 \pi / 2 \leq 0$

$g_{9}(X)=r_{1} \cos \theta_{1}+r_{4} \cos \theta_{4}-r_{3} \cos \theta_{3}-r_{21} \cos \theta_{2}=0$ (14)

$g_{10}(X)=r_{1} \sin \theta_{1}+r_{4} \sin \theta_{4}-r_{3} \sin \theta_{3}-r_{21} \sin \theta_{2}=0$

$g_{11}(X)=r_{22} \cos \theta_{2}+r_{6} \cos \theta_{6}-r_{7} \cos \theta_{7}-r_{3} \cos \theta_{3}-r_{5} \cos \theta_{5}=0$

$g_{12}(X)=r_{22} \sin \theta_{2}+r_{6} \sin \theta_{6}-r_{7} \sin \theta_{7}-r_{3} \sin \theta_{3}-r_{5} \sin \theta_{5}=0$

The variable $X$ and it's upper/lower bounds are (Eqs. 18-21):

$0 \mathrm{~cm} \leq X_{1} \leq 20 \mathrm{~cm}$
$0 \mathrm{~cm} \leq X_{2} \leq 20 \mathrm{~cm}$
$0 \mathrm{~cm} \leq X_{3} \leq 20 \mathrm{~cm}$
$0 \mathrm{~cm} \leq X_{4} \leq 20 \mathrm{~cm}$

In practical application, the patient's $X$ values are input to the above equations. The "seqential quadratic programming method" [10] is used to obtain the optimum solutions, and the results are used to fabricate a customized SWIMS splint for the patient. Five experimental Type I SWIMS splints were constructed. The actual joint angles generated by the prototypes will be compared with the analytical solutions in a following section.

\section{EXPERIMENT}

Five normal subjects, male, average age 25 , with no physical abnormalities, were recruited from the students at National Cheng Kung University Medical Center. From their physical measurements, five prototype SWIMS Type I dynamic splints were fabricated, one for each subject, each one custom-made for one subject.

Each subject wore his splint during experiments wherein the relative joint positions of the hand and wrist were measured by a six-camera threedimentional motion analysis system. Passive external reflective makers were placed on the dorsal surface of the subject's hand. The positions of the markers are shown in Fig. 3. During the experiment, the subject's hand is relaxed and only the wrist has flexion and extension. The experimental motion cycle is defined as follows: when the wrist flexes, the fingers extend, and when the wrist extends, the fingers flex. For kinetic analysis, each finger segment is assumed to be a rigid body, the PIP joint is assumed to be a hinge joint, and only flexion and extension are allowed. The MP joint and wrist joint are simplified into a single hinge joint. The mathematical models of the instantaneous angles for the PIP joint, MP joint and wrist joint are:

1. PIP joint angle (Eqs. 22):

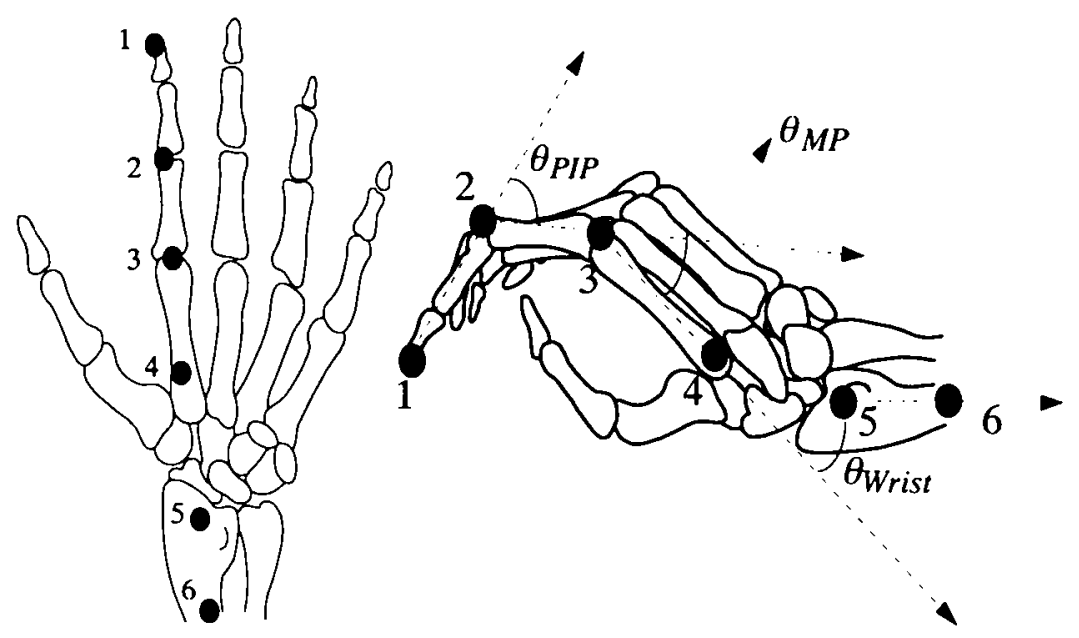

Fig 3. Marker set for hand motion analysis and picture of Wrist, MP and PIP joints. 
Table 1: Finger and palm length of subjects

\begin{tabular}{||c|c|c|c|c|c|c||}
\hline & $r_{1}$ & $r_{3}$ & $r_{4}$ & $r_{5}$ & $\theta_{1}$ & $\beta$ \\
\hline subject 1 & $3.5277 \mathrm{~cm}$ & $6.4422 \mathrm{~cm}$ & $5.2604 \mathrm{~cm}$ & $15.2355 \mathrm{~cm}$ & $\frac{\pi}{3}$ & $-\frac{\pi}{15}$ \\
\hline subject 2 & $2.8658 \mathrm{~cm}$ & $4.7563 \mathrm{~cm}$ & $7.6272 \mathrm{~cm}$ & $14.8900 \mathrm{~cm}$ & $\frac{10 \pi}{21}$ & $\frac{\pi}{60}$ \\
\hline subject 3 & $3.0976 \mathrm{~cm}$ & $5.2120 \mathrm{~cm}$ & $6.8117 \mathrm{~cm}$ & $14.1473 \mathrm{~cm}$ & $\frac{100 \pi}{245}$ & 0 \\
\hline subject 4 & $2.4235 \mathrm{~cm}$ & $4.6691 \mathrm{~cm}$ & $6.1470 \mathrm{~cm}$ & $14.0208 \mathrm{~cm}$ & $\frac{10 \pi}{26}$ & $-\frac{\pi}{15}$ \\
\hline subject 5 & $3.2457 \mathrm{~cm}$ & $5.3707 \mathrm{~cm}$ & $7.4425 \mathrm{~cm}$ & $14.6304 \mathrm{~cm}$ & $\frac{10 \pi}{22}$ & $\frac{\pi}{60}$ \\
\hline
\end{tabular}

Table 2: Result under control five points

\begin{tabular}{||c|c|c|c|c|c|c||}
\hline & $r_{1}$ & $r_{3}$ & $r_{4}$ & $r_{5}$ & $\theta_{1}$ & $\beta$ \\
\hline subject 1 & $3.5277 \mathrm{~cm}$ & $6.4422 \mathrm{~cm}$ & $5.2604 \mathrm{~cm}$ & $15.2355 \mathrm{~cm}$ & $\frac{\pi}{3}$ & $-\frac{\pi}{15}$ \\
\hline subject 2 & $2.8658 \mathrm{~cm}$ & $4.7563 \mathrm{~cm}$ & $7.6272 \mathrm{~cm}$ & $14.8900 \mathrm{~cm}$ & $\frac{10 \pi}{21}$ & $\frac{\pi}{60}$ \\
\hline subject 3 & $3.0976 \mathrm{~cm}$ & $5.2120 \mathrm{~cm}$ & $6.8117 \mathrm{~cm}$ & $14.1473 \mathrm{~cm}$ & $\frac{100 \pi}{245}$ & 0 \\
\hline subject 4 & $2.4235 \mathrm{~cm}$ & $4.6691 \mathrm{~cm}$ & $6.1470 \mathrm{~cm}$ & $14.0208 \mathrm{~cm}$ & $\frac{10 \pi}{26}$ & $-\frac{\pi}{15}$ \\
\hline subject 5 & $3.2457 \mathrm{~cm}$ & $5.3707 \mathrm{~cm}$ & $7.4425 \mathrm{~cm}$ & $14.6304 \mathrm{~cm}$ & $\frac{10 \pi}{22}$ & $\frac{\pi}{60}$ \\
\hline
\end{tabular}

Table 3: Results using eight control points

\begin{tabular}{||c|c|c|c|c|c|c||}
\hline & $r_{1}$ & $r_{3}$ & $r_{4}$ & $r_{5}$ & $\theta_{1}$ & $\beta$ \\
\hline subject 1 & $3.2585 \mathrm{~cm}$ & $6.0350 \mathrm{~cm}$ & $5.4366 \mathrm{~cm}$ & $14.8689 \mathrm{~cm}$ & $\frac{\pi}{3}$ & $-\frac{\pi}{15}$ \\
\hline subject 2 & $3.2135 \mathrm{~cm}$ & $5.4197 \mathrm{~cm}$ & $8.0027 \mathrm{~cm}$ & $15.5421 \mathrm{~cm}$ & $\frac{10 \pi}{21}$ & $\frac{\pi}{30}$ \\
\hline subject 3 & $3.0001 \mathrm{~cm}$ & $5.1472 \mathrm{~cm}$ & $6.9006 \mathrm{~cm}$ & $14.0995 \mathrm{~cm}$ & $\frac{10 \pi}{25}$ & 0 \\
\hline subject 4 & $3.3790 \mathrm{~cm}$ & $5.9707 \mathrm{~cm}$ & $6.6944 \mathrm{~cm}$ & $15.4448 \mathrm{~cm}$ & $\frac{10 \pi}{28}$ & $-\frac{\pi}{30}$ \\
\hline subject 5 & $2.9025 \mathrm{~cm}$ & $4.8813 \mathrm{~cm}$ & $7.4454 \mathrm{~cm}$ & $14.1672 \mathrm{~cm}$ & $\frac{10 \pi}{22}$ & $\frac{\pi}{30}$ \\
\hline
\end{tabular}

$$
\theta_{P I P}=\cos ^{-1}\left(\frac{\overrightarrow{p_{1} p_{2}} \cdot \overrightarrow{p_{2} p_{3}}}{\left|\overrightarrow{p_{1} p_{2}} \| \overrightarrow{p_{2} p_{3}}\right|}\right)
$$

2. MP joint angle (Eqs. 23):

$$
\theta_{M P}=\cos ^{-1}\left(\frac{\overrightarrow{p_{2} p_{3}} \cdot \overrightarrow{p_{3} p_{4}}}{\left.\mid \overrightarrow{p_{2} p_{3}|| \overrightarrow{p_{3} p_{4}} \mid}\right)}\right)
$$

3. wrist joint angle (Eqs. 24): 

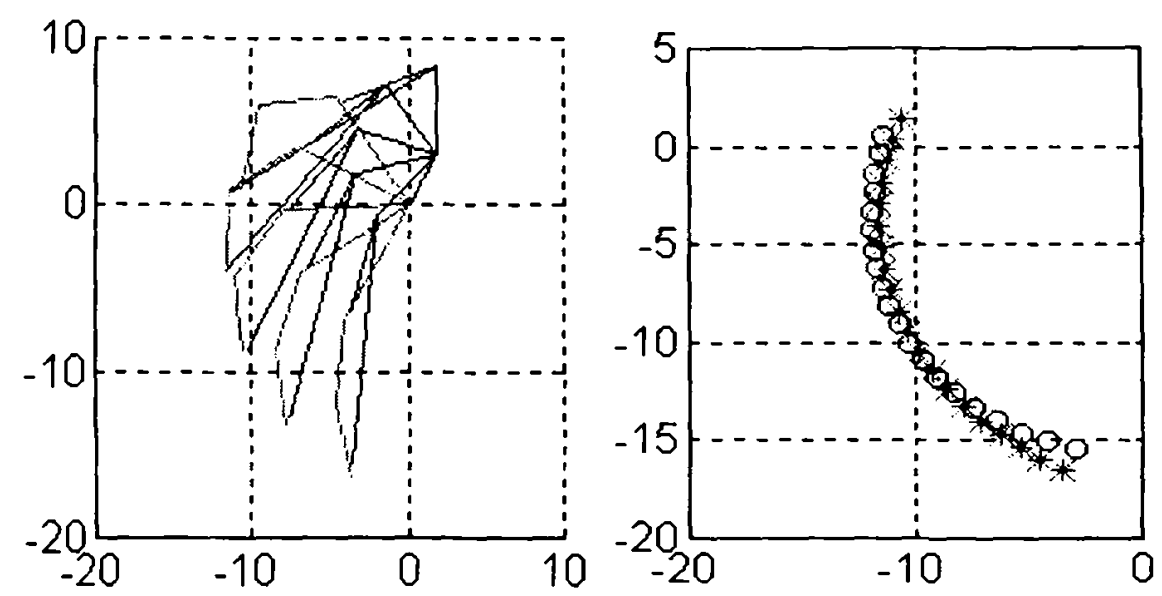

Fig. 4: Motion of $D$ point (with five control points)
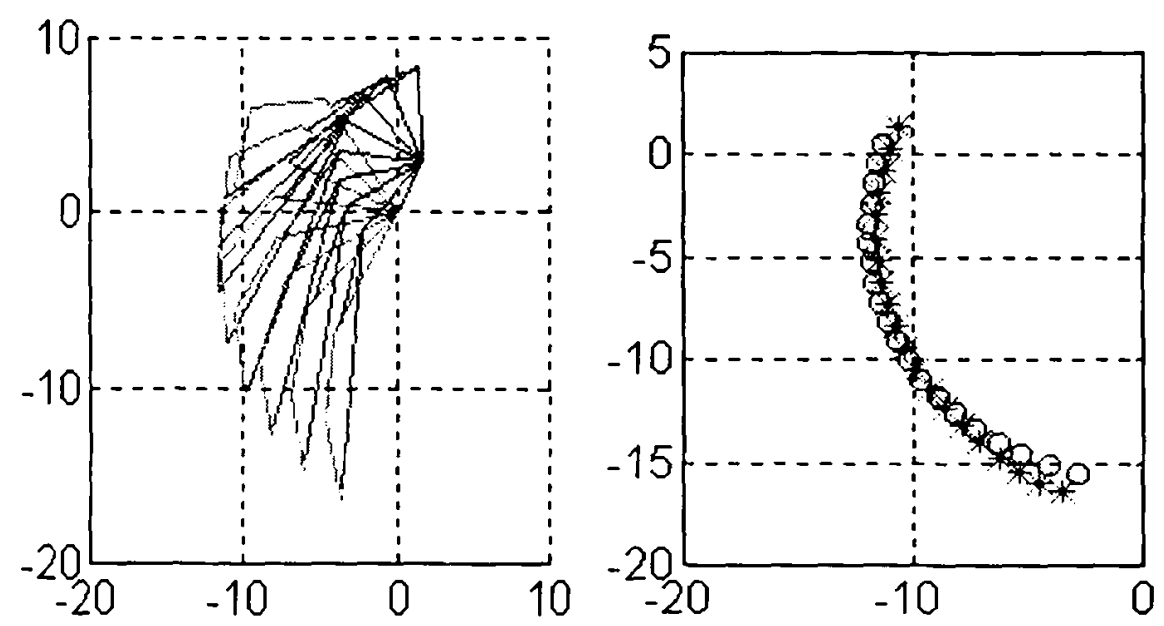

Fig. 5 : Motion of $D$ point (with eight control points)

$$
\theta_{\text {Wrist }}=\cos ^{-1}\left(\underset{\overrightarrow{p_{3} p_{4}} \cdot \overrightarrow{p_{5} p_{6}}}{\overrightarrow{\overrightarrow{p_{3} p_{4}}} \| \overrightarrow{p_{5} p_{6}}}\right)
$$

\section{RESULTS}

The measurements for each segment of the subject's index finger (wrist joint to MP joint, MP joint to PIP joint and PIP joint to TIP) are used with optimization methods to determine suitable crank lengths $r 1, r 3$, $\mathrm{r} 4, \mathrm{r} 5$ and angles $\theta 1$ and $\beta$ for splint manufacture. The motion curves predicted numerically for point $\mathrm{D}$ (TIP) are then compared with the motion curves observed by the six-camera motion system for Type I
SWIMS. Table 1 shows the finger segment lengths. Tables $2 \& 3$ show the optimized dimensions, Table 2 using 5 control points, Table 3 using 8 control points. The results in Table 2 are presented graphically in Fig. 4 as the curve composed of zeros, showing the numerically generated motion of point D. Fig 6 also shows, as the curve composed of asterisks, the TIP curve of the hand as measured by the six-camera system. Similar data is shown in Table 3 and Fig. 5 for the eight-control-point results. As can be seen, both the 5 and 8 point simulations provide approximately equal results. Thus, 5-control-point optimization may be used for splint design.

\section{DISCUSSIONS AND CONCLUSION}

It is desired to fit the orbit of point $\mathrm{D}$ to the orbit 


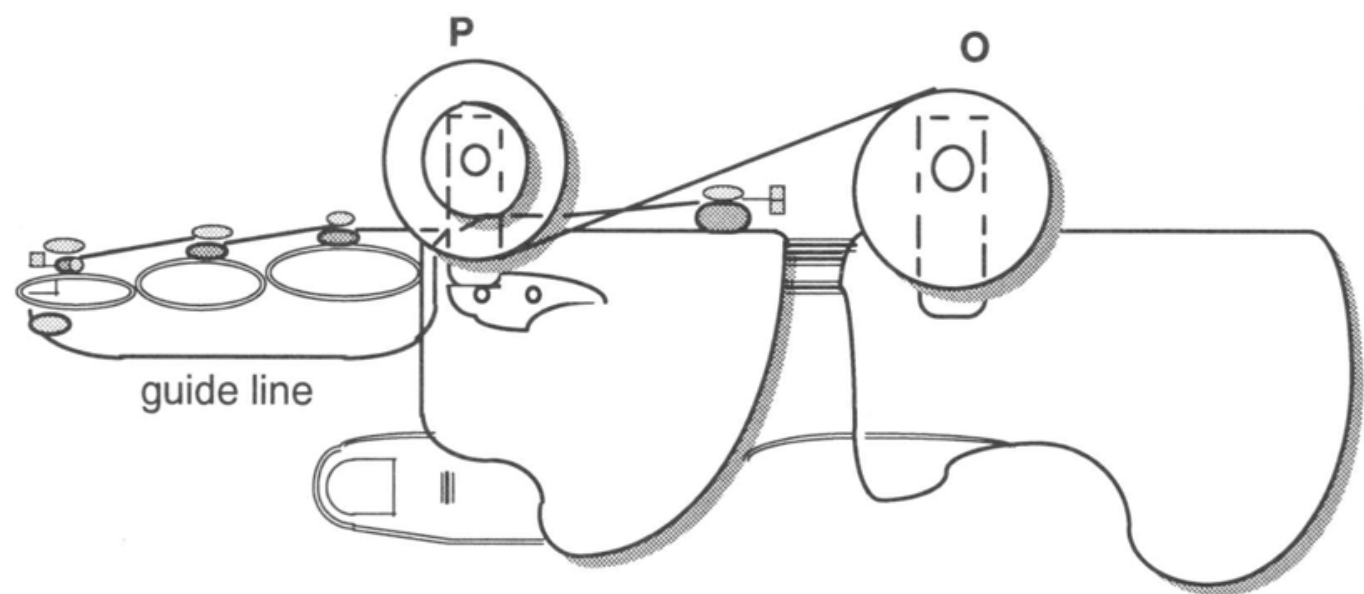

Fig. 6: Lateral view of Type II dynamic splint

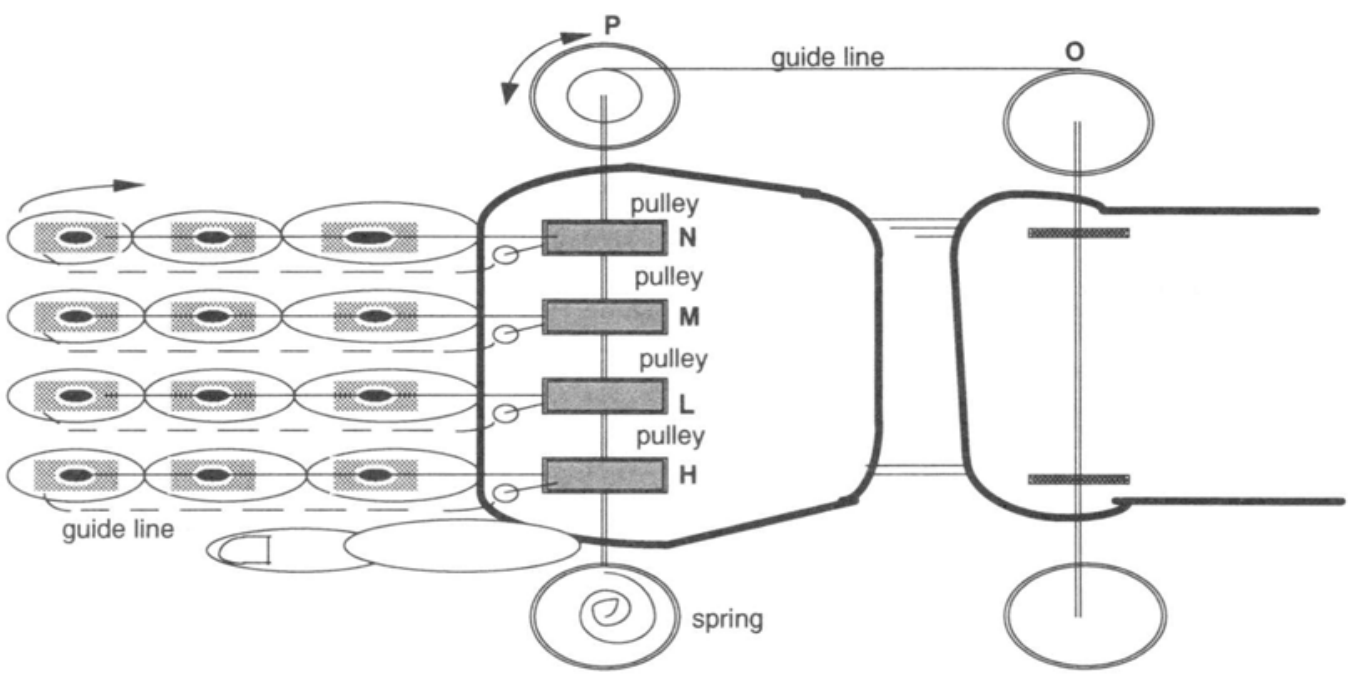

Fig. 7: Upper view of Type II dynamic splint

of TIP for the range of natural wrist motion by optimization of input values for angles $\theta_{1}$ and $\beta$ and ratios $r_{21}$ and $r_{22}$. For most of the orbit of the Type I SWIMS prototype, D and TIP curves were similar, with modcratcly increased differences at the beginning and end of the curves. Thus, it can be seen that Watt's six-bar linkage mechanism can be used to successfully simulate wrist motion, and, further, that Type I SWIMS would seem useful in providing optimal dynamic splinting for hand surgery patients. Final confirmation of Type I SWIMS, however, awaits clinical testing.

Our main discovers are listed as below :

1.Watt's six-bar linkage mechanism provides a useful basis for design of a dynamic wrist splint.

2.Comparison of the experimentally-observed motion of a Type I SWIMS prototype with the numerical model shows good agreement between obtained and desired results for wrist flexion and extension less than
40 degrees.

3.It was found that the results for both 5 and 8 points simulations provided approximately equal results. Thus, 5-control-point optimization may be used for splint design.

\section{FUTURE}

Clinical testing of the Type I SWIMS is of interest. A pulley-based Type II SWIMS design is in progress (Fig.6,7). We are developing a general model for the relationship between each segment of the fingers. From this, it would be possible to determine the crank length needed to fabricate the dynamic splint simply and directly from a patient's finger length.

Because the lengths of linkages 5 and 6 are equal to the lengths from MP joint to PIP joint and from PIP joint to TIP joint, respectively, it may be possible to 
simplify the model into four-bar linkage mechanism. However, the motion center axis of each segment of the palm must be measured before analyzing this mechanism.

\section{REFERENCES}

1. Bunnell S. Surgery of the Hand Philadephia, J. B. Lippincott 1944; 340-343.

2. Mason ML, Allen HS. The rate of heailing of tendons-anexperimental study of tensile strength. Ann Surg 1941; 113:424.

3 Young RES, Harmon JM. Repair of tendon injuries of the hand. Ann Surg 1960;151:562.

4 Gelberman RH, Woo SLY et al. Effects of early intermittent passive mobilization on healing canine flexor tendons, J.Hand Surgery 1982;7170-175.

5. Hitchcock TF, Light TR, Bunch WH et al. The effect of immediate controlled mobilization on the strength of flexor tendor repair. Trans Orthopedic
Research Society 1986; 11:216.

6. Chow JA, Thomas LJ, Provost JM, et al,. A combined regimen of controlled motion following flexor tendor repair in "No Man's Land". Plastic And Reconstructive Surgery 1987;79:447-453.

7. Kleinert HE, Kutz JE, Cohen MJ. Primary repair of zone II flexor tendon lacerations. In AAOS Symposium on tendon Surgery in the Hand. St Louis, C. V. Mosby 1975;91-104.

8 Lister GD, Klieinert HE, Kutz JE, Atasoy E. Primary flexor repair by immediate controlled mobilization. J. Hand Surg. 1977;2:441.

9 Cooney WP, Lin GT, An KN. Improve tendod excursion fllowing flexor tendon repair,J. Hand Therapy, 1989;102:106.

10 Garret N. Vanderplaats: Numerical optimization techniques for enginnering desing: with applications.1984,Publisher McGraw-Hill Inc.

11. Gibdon CG, D. Marsh. On the Linkage Varieties of Watt 6-Bat Mechanisms-I. Basic Geomery. Mech. Mach. Theory 1989;24:105-113. 